\title{
Dementia with Lewy bodies: a comparison of clinical diagnosis, FP-CIT single photon emission computed tomography imaging and autopsy
}

\section{Zuzana Walker, Evelyn Jaros, Rodney W H Walker, Lean Lee, Durval C Costa, Gill Livingston, Paul G Ince, Robert Perry, lan McKeith, Cornelius L E Katona}

J Neurol Neurosurg Psychiatry 2007;78:1176-1 181. doi: 10.1136/jnnp.2006.110122

See end of article for authors' affiliations

Correspondence to: Dr Zuzana Walker Department of Mental Health Sciences, St Margaret's Hospital, Epping, Essex, UK; z.walker@ucl.ac.uk

Received 29 October 2006 Revised 18 February 2007 Accepted 23 February 2007 Published Online First 12 March 2007
Background: Dementia with Lewy bodies (DLB) is a common form of dementia. The presence of Alzheimer's disease (AD) pathology modifies the clinical features of DLB, making it harder to distinguish DLB from AD clinically during life. Clinical diagnostic criteria for DLB applied at presentation can fail to identify up to $50 \%$ of cases. Our aim was to determine, in a series of patients with dementia in whom autopsy confirmation of diagnosis was available, whether functional imaging of the nigrostriatal pathway improves the accuracy of diagnosis compared with diagnosis by means of clinical criteria alone.

Methods: A single photon emission computed tomography (SPECT) scan was carried out with a dopaminergic presynaptic ligand [ ${ }^{123}$ I]-2beta-carbometoxy-3beta-(4-iodophenyl)-N-(3-fluoropropyl) nortropane (FP-CIT; ioflupane) on a group of patients with a clinical diagnosis of DLB or other dementia. An abnormal scan was defined as one in which right and left posterior putamen binding, measured semiquantitatively, was more than 2 SDs below the mean of the controls.

Results: Over a 10 year period it was possible to collect 20 patients who had been followed from the time of first assessment and time of scan through to death and subsequent detailed neuropathological autopsy. Eight patients fulfilled neuropathological diagnostic criteria for DLB. Nine patients had AD, mostly with coexisting cerebrovascular disease. Three patients had other diagnoses. The sensitivity of an initial clinical diagnosis of DLB was $75 \%$ and specificity was $42 \%$. The sensitivity of the FP-CIT scan for the diagnosis of DLB was $88 \%$ and specificity was $100 \%$.

Conclusion: FP-CIT SPECT scans substantially enhanced the accuracy of diagnosis of DLB by comparison with clinical criteria alone.
D istinguishing patients with dementia with Lewy bodies (DLB) from those with Alzheimer's disease (AD) can be challenging, in some cases even after repeated follow-up observation. Imaging modalities which assess the integrity of the nigrostriatal pathway have detected impaired function of this pathway in patients with clinically diagnosed DLB but preserved function in patients with clinically diagnosed AD. ${ }^{1-8}$ Such imaging is potentially of considerable value in supporting a diagnosis of DLB. However, the accuracy of the clinical diagnosis of DLB is imperfect, ${ }^{9}{ }^{10}$ meaning that estimation of the diagnostic accuracy of the imaging investigation needs to be done against neuropathological findings.

We have been following a cohort of patients with dementia whose initial dementia diagnosis was made on the basis of standard clinical criteria (for $\mathrm{AD}^{11}{ }^{11}$ for $\mathrm{DLB}^{12}$ ). These patients underwent a single photon emission computed tomography (SPECT) scan using $\left[{ }^{123} \mathrm{I}\right]$-2beta-carbometoxy-3beta-(4-iodophenyl)-N-(3-fluoropropyl) nortropane (FP-CIT; ioflupane), a ligand that binds to the presynaptic dopamine transporter, demonstrating the integrity of the nigrostriatal projection pathway. Scanning was undertaken at the time of presentation to our service-that is, directly after the clinical diagnosis was made. Autopsies are now available for 20 patients, enabling us to report what abnormal FP-CIT scans actually equate to in pathological terms. Furthermore, this autopsy series allows a more robust assessment of the role of FP-CIT SPECT scanning in the diagnosis of DLB than can be made using clinical diagnoses only.

\section{METHODS}

Sixty-one clinically diagnosed patients and controls (17 AD, 27 DLB, 1 patient with cortico-basal degeneration (CBD) and 16 controls) were scanned with dopaminergic presynaptic ligand FP-CIT SPECT between 1996 and 1999. All patients were then prospectively followed-up. Of the original 45 patients with dementia, 30 have died (67\%) and in 22/30 cases the family agreed to autopsy examination (73.3\% autopsy rate). Here we present the results of the first 20 patients with dementia in whom comprehensive brain autopsy examination is available.

Detailed descriptions of the clinically diagnosed AD and DLB patients, and the imaging results of the original clinical cohort, have been published previously. ${ }^{5}$ At that time, autopsy results were available for 10 of the cases and were reported briefly. In the original cohort, patients with dementia were ascribed to either the DLB or AD group on the basis of fulfilling the Consensus DLB criteria ${ }^{12}$ or NINCDS-ADRDA criteria. ${ }^{11}$ Many of the patients with DLB fulfilled both sets of criteria, and these patients were classified as having DLB. In addition, in the series reported here, we have included one further patient who was diagnosed clinically as having CBD but nevertheless had a FPCIT SPECT scan and was followed prospectively and then underwent autopsy. Sixteen healthy elderly controls ( 10 males;

Abbreviations: $A D$, Alzheimer's disease; $C B D$, cortico-basal degeneration; CVD, cerebrovascular disease; DLB, dementia with Lewy bodies; LB, Lewy body; NFT, neurofibrillary tangle; PD, Parkinson's disease; PET, positron emission tomography; SMU, Strichman Medical Unit; SPECT, single photon emission computed tomography 
6 females; mean age 67 years) were recruited from relatives and partners of patients (mainly spouses). Their mean Unified Parkinson's Disease Rating Scale score was 2.2, mean MiniMental State Examination score was 28.9 and mean Cambridge Cognitive Examination score was 102.

\section{FP-CIT SPECT scan}

All subjects underwent scanning with a brain dedicated scanner, the Strichman Medical Equipment 810 linked to a Macintosh computer. The Strichman camera consists of 12 individual detectors, each equipped with a focusing collimator. The transaxial resolution of this camera is $7.6 \mathrm{~mm}$ full width half maximum and axial resolution is $12.5 \mathrm{~mm}$. The measured concentration of radioactivity was expressed as Strichman Medical Units (SMUs; I SMU $=100 \mathrm{~Bq} / \mathrm{ml}$ ). Scanning took place between 3 and $4 \mathrm{~h}$ after injection of FP-CIT (DaTSCAN) (150-185 MBq). Usually 8-10 slices were acquired starting at the cerebellum level upwards to include the basal ganglia. The overall scanning time for each patient was 30-45 min.

Two patients were taking levodopa medication and three patients were taking neuroleptic medication at the time of scanning. One patient took sulpiride $200 \mathrm{mg}$ at night and two patients took olanzapine $5 \mathrm{mg}$ /day. These medications were not discontinued because they do not interfere with dopamine transporter imaging. ${ }^{13-16}$

\section{Semiquantitative analysis of scans}

DCC, who was blinded to the clinical and autopsy diagnostic status of all subjects, performed a semiquantitative analysis of all scans. The images were reconstructed automatically. For the analysis of striatal binding, the ratio of specific to non-specific binding was calculated by summing the two adjacent transverse slices that demonstrated the most intense striatal uptake. Identical circular regions of interest were used to calculate the average striatal (the caudate nucleus, the anterior and the posterior putamen) to non-specific (the occipital cortex as a region with little or no dopamine receptors) radioactivity ratios for both hemispheres. The formula used was:

FP-CIT binding $=$ STR/OCC

where STR is the mean radioactivity (in SMU) in the striatum (caudate, anterior and posterior putamen) and OCC is the mean radioactivity in the occipital cortex.

An abnormal scan, signifying a more likely diagnosis of DLB, was defined as a scan with semiquantitative binding in the

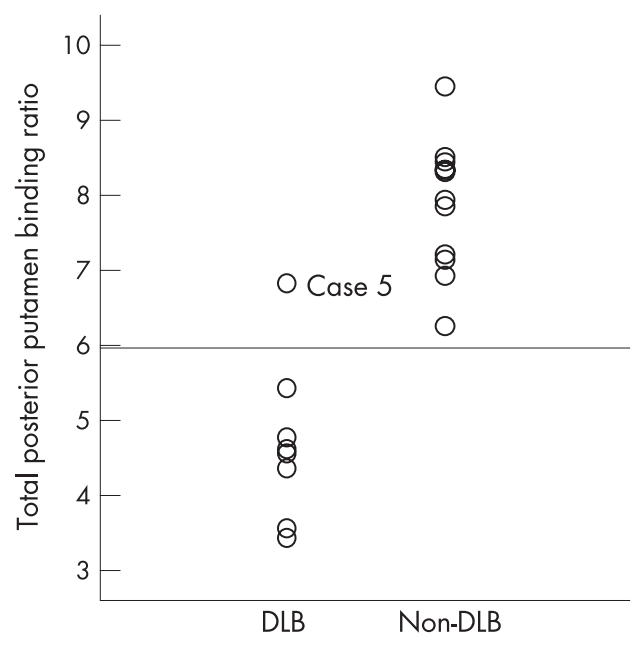

Figure 1 Total (right and left) posterior putamen radioactivity ratios for cases of dementia with Lewy bodies (DLB) and other dementia cases. The horizontal line on the graph marks the value (5.95) for 2 SDs below the mean of the total posterior putamen binding ratios of the controls. posterior putamen (right and left), which was more than 2 SDs below the mean of the controls. In the controls, the mean values for left posterior putamen binding ratio and right posterior putamen binding ratio were 4.43 and 4.44 , respectively. The mean for left plus right posterior putamen binding ratio was 8.87 (SD 1.46). Thus the mean minus 2 SDs was 5.95 (fig 1). We also present the results based on defining an abnormal scan as having posterior putamen binding on just one side (either right or left) more than 2 SDs below the mean of the controls (ie, <2.91).

\section{Visual rating of scans}

As a separate exercise, all scans were presented randomly and assessed visually by three independent raters (DCC, ZW and RWHW) experienced in assessing FP-CIT scans, who were blinded to the clinical and autopsy diagnoses. Scans were scored as follows: normal uptake in all regions (right and left caudate and right and left putamen) $=0$; slight reduction in uptake in any of the four regions $=1$; and significant reduction in uptake in any of the four regions $=2$. Subsequently, for all statistical analyses, scans with scores of 0 or 1 were combined into a "normal" group and scans with a score 2 were declared "abnormal".

\section{Neuropathological examination}

Detailed neuropathological examination was performed in Newcastle by RP, PI and EJ.

\section{Brain areas sampled}

At autopsy, the left cerebral and cerebellar hemispheres were dissected and snap frozen for neurochemical examination. The right half of the brain was fixed in formalin, coronally sliced at $7 \mathrm{~mm}$ intervals, and cortical, subcortical and brainstem areas were sampled and embedded in paraffin to establish the neuropathological diagnosis. The brain areas sampled included the entorhinal cortex at the level of the uncus; hippocampus and medial temporal cortex at the level of the geniculate bodies; anterior cingulate cortex; and cerebral neocortex and adjoining deep white matter at the pre-frontal, mid-frontal, posterior frontal, posterior lateral parietal, lateral temporal (at the level of the geniculate bodies) and the posterior occipital levels. The subcortical nuclei sampled included the amygdala, striatum, globus pallidus, thalamus and subthalamic nucleus. In the cerebellum, the vermis, medial and lateral cerebellar hemisphere and dentate nucleus were sampled. The brainstem nuclei sampled included the substantia nigra at the level of the red nucleus and the decussation of the cerebellar peduncle, pedunculo-pontine nucleus, dorsal raphe nucleus, locus

Table 1 Clinical characteristics of the neuropathologically diagnosed patients with and without dementia with Lewy bodies

\begin{tabular}{lrc}
\hline Characteristic & $\begin{array}{l}\text { DLB (\%) } \\
(\mathbf{n}=8)\end{array}$ & $\begin{array}{l}\text { Non-DLB (\%) } \\
(\mathbf{n}=12)\end{array}$ \\
\hline Positive family history of dementia & $2(25)$ & $4(33)$ \\
Past psychiatric history & $0(0)$ & $2(17)$ \\
Acute confusional states & $2(25)$ & $0(0)$ \\
Rigidity & $5(63)$ & $4(33)$ \\
Akinesia/bradykinesia & $5(63)$ & $5(42)$ \\
Tremor & $4(50)$ & $8(67)$ \\
Fluctuating course of illness & $6(75)$ & $7(58)$ \\
Visual hallucinations & & \\
Once only & $2(25)$ & $1(8)$ \\
Persistent & $4(50)$ & $5(42)$ \\
\hline
\end{tabular}

DLB, dementia with Lewy bodies.

There were no significant differences between the DLB and non-DLB groups. 
Table 2 Demographic characteristics and test scores of neuropathologically diagnosed patients with and without dementia with Lewy bodies

\begin{tabular}{lll}
\hline Characteristic & $\begin{array}{l}\text { DLB } \\
\text { (n=8) }\end{array}$ & $\begin{array}{l}\text { Non-DLB } \\
(\mathbf{n}=12)\end{array}$ \\
\hline Sex ratio (M:F) & 3.5 & $3: 9$ \\
Age at onset of dementia (y) & $73.3(9)$ & $73.2(10)$ \\
Age at time of FP-CIT (y) & $76.5(9)$ & $77.8(9)$ \\
Time from scan to death (months) & $34(22)$ & $34(26)$ \\
Years of education & $9.5(1.4)$ & $10.4(1.9)$ \\
H\&Y stage & $1.9(1.5)$ & $1.4(1.5)$ \\
UPDRS score & $18.1(13.8)$ & $13.0(12.7)$ \\
MMSE & $17(5.6)$ & $16.6(8.8)$ \\
CAMCOG & $60(21)$ & $41(27)$ \\
CAPE & $9.4(7)$ & $12.3(9)$ \\
GDS & $3.9(3)$ & $3.3(2)$ \\
CDR & $1.4(0.9)$ & $1.7(0.9)$ \\
Behave-AD & $10.3(7)$ & $10.3(8)$ \\
\hline
\end{tabular}

Behave-AD, behavioural pathology in Alzheimer's disease; CAMCOG, Cambridge Cognitive Examination; CAPE, Cliffon Assessment Procedure for the Elderly; CDR, Clinical Dementia Rating; DLB, dementia with Lewy bodies; GDS, Geriatric Depression Scale; H\&Y stage, Hoehn and Yahr stage; MMSE, Mini-Mental State Examination; UPDRS, Unified Parkinson's Disease Rating Scale (motor section).

Values are mean (SD).

There were no significant differences between the DLB and non-DLB groups.

coeruleus, pontine nuclei, dorsal nucleus of vagus, reticular formation and inferior olive.

\section{Neurohistology}

Paraffin sections $(6 \mu \mathrm{m})$ from the cortical, subcortical and brainstem areas were examined using standard neuropathological stains, including haematoxylin and eosin for general histology, cresyl fast violet for neuronal population density, Loyez for myelin, Gallyas or methenamine silver stains and AT8 (monoclonal antibodies to the tau protein phosphorylated at Ser-202; 90206 (BR-003) Innogenetics antibodies from AutogenBioclear UK Ltd, Calne, Wiltshire, UK) for neuritic plaques, Gallyas for neurofibrillary tangles (NFTs) and ghost tangles, AT8 for pre-tangles and NFTs, $\alpha$-synuclein (monoclonal antibodies recognising an epitope of the protein at AA 160; NCL-ASYN Novocastra antibodies from VisionBiosystems Europe Ltd, Newcastle upon Tyne, UK) for Lewy bodies (LBs), pre-LBs and neurites, ubiquitin (polyclonal antibodies Z0458 from Dako UK Ltd, Ely, Cambridgeshire, UK) for LBs in some cases and $\beta A 4$ antibodies (monoclonal M0872 from Dako UK Ltd) for amyloid angiopathy and diffuse and plaque associated amyloid.

\section{Neuropathological diagnostic criteria}

The neuropathological diagnostic criteria employed for $\mathrm{AD}$ included the following: CERAD (Consortium to Establish a Registry for Alzheimer's Disease) score and diagnosis, ${ }^{17}$ Braak stage $^{18}$ and NIA-RI (National Institute on Aging and Reagan Institute) $\mathrm{AD}$ diagnosis. ${ }^{19}$ In addition, based on experience at Newcastle, ${ }^{20}$ NFTs were counted in the temporal, frontal, parietal and occipital neocortex and their densities (number per $\mathrm{mm}^{2}$ ) were used to stage the neocortical NFT pathology as follows: none $=$ no NFTs in the neocortex; not significant $=$ fewer than 4 NFTs per $\mathrm{mm}^{2}$ in each of the four lobes; moderate $=$ fewer than 4 NFTs per $\mathrm{mm}^{2}$ in one or more lobes but mean density from all the four lobes of more than 4 NFTs per $\mathrm{mm}^{2}$; significant $=$ more than 4 NFTs per $\mathrm{mm}^{2}$ in each of the four lobes.

The neuropathological diagnostic criteria employed for DLB were those recommended by the Third report of the DLB Consortium. ${ }^{21}$

\section{Statistics}

Data were analysed using SPSS/PC+ version 12.0.1 (Statistical Package for Social Sciences). Taking the autopsy diagnosis to be the "true" or "gold standard" diagnosis, we calculated the sensitivity and specificity of FP-CIT SPECT and the sensitivity and specificity of the Consensus DLB criteria (1996) applied at the initial clinical assessment for making the diagnosis of DLB.

Table 3 Initial clinical, imaging and autopsy diagnosis

\begin{tabular}{|c|c|c|c|c|c|}
\hline $\begin{array}{l}\text { Case } \\
\text { No }\end{array}$ & $\begin{array}{l}\text { Clinical } \\
\text { diagnosis }\end{array}$ & $\begin{array}{l}\text { No of core } \\
\text { features of } \\
\text { DLB }\end{array}$ & $\begin{array}{l}\text { Visual } \\
\text { rating } \\
\text { diagnosis }\end{array}$ & $\begin{array}{l}\text { Semiquantitative } \\
\text { diagnosis }\end{array}$ & $\begin{array}{l}\text { Neuropathological } \\
\text { diagnosis }\end{array}$ \\
\hline 1 & $A D$ & 0 & Abn & Abn & $D L B+A D$ \\
\hline 2 & DLB & 3 & Abn & Abn & DLB \\
\hline 3 & DLB & 2 & $\mathrm{~N}$ & Abn & DLB \\
\hline 4 & DLB & 2 & Abn & $A b n$ & $\mathrm{DLB}+\mathrm{AD}+\mathrm{CVD}$ \\
\hline 5 & CBD & 1 & Abn & $\mathrm{N}$ & $D L B+A D$ \\
\hline 6 & DLB & 2 & Abn & Abn & DLB \\
\hline 7 & DLB & 2 & Abn & Abn & $\mathrm{DLB}+\mathrm{CVD}$ \\
\hline 8 & DLB & 3 & Abn & Abn & DLB \\
\hline 9 & $A D$ & 0 & $\mathrm{~N}$ & $\mathrm{~N}$ & $A D+C V D$ \\
\hline 10 & $A D$ & 0 & $\mathrm{~N}$ & $\mathrm{~N}$ & $A D+C V D+m e t a s t a t i c ~ c a r c i n o m a$ \\
\hline 11 & DLB & 2 & Abn & $\mathrm{N}$ & $A D+C V D$ \\
\hline 12 & DLB & 2 & $\mathrm{~N}$ & $\mathrm{~N}$ & $A D$ \\
\hline 13 & DLB & 2 & $\mathrm{~N}$ & $\mathrm{~N}$ & CBD \\
\hline 14 & DLB & 2 & $\mathrm{~N}$ & $\mathrm{~N}$ & $A D$ \\
\hline 15 & $A D$ & $\overline{1}$ & $\mathrm{~N}$ & $\mathrm{~N}$ & Unspecified pathology \\
\hline 16 & $A D$ & 0 & N & $\mathrm{N}$ & $A D+C V D$ \\
\hline 17 & DLB & 1 & Abn & $\mathrm{N}$ & FTLD \\
\hline 18 & DLB & 2 & $\mathrm{~N}$ & $\mathrm{~N}$ & $A D$ \\
\hline 19 & DLB & 2 & $\mathrm{~N}$ & $\mathrm{~N}$ & $A D+C V D$ \\
\hline 20 & $A D$ & 0 & $\mathrm{~N}$ & $\mathrm{~N}$ & $A D+C V D$ \\
\hline
\end{tabular}

Abn, abnormal scan; AD, Alzheimer's disease; CBD, cortico-basal degeneration; CVD, cerebrovascular disease; DLB, dementia with Lewy bodies; FTLD, fronto-temporal lobar degeneration; N, normal scan.

In the visual rating there was complete agreement between all three raters for 15 cases. In the remaining five cases there was a 2:1 split. In two of these cases the dissent related to scores of 0 or 1 , therefore having no effect on the assignment of the scan as normal or abnormal. The three cases where disagreement potentially affected the assignment of the scan were case Nos 1, 7 and 12 . 
Table 4 Neuropathological diagnosis and diagnostic criteria

\begin{tabular}{|c|c|c|c|c|c|c|c|c|c|c|}
\hline $\begin{array}{l}\text { Case } \\
\text { No }\end{array}$ & $\begin{array}{l}\text { EPF } \\
\text { at time } \\
\text { of scan }\end{array}$ & $\begin{array}{l}\text { Clinical } \\
\text { diagnosis }\end{array}$ & $\begin{array}{l}\text { Neuropathological } \\
\text { diagnosis }\end{array}$ & $\begin{array}{l}\text { Braak } \\
\text { stage }\end{array}$ & $\begin{array}{l}\text { Neocortical } \\
\text { NFT stage* }\end{array}$ & $\begin{array}{l}\text { CERAD } \\
\text { score }\end{array}$ & $\begin{array}{l}\text { AD } \\
\text { diagnosis } \\
\text { by CERAD } \\
\text { criteria }\end{array}$ & $\begin{array}{l}\text { AD diagnosis } \\
\text { by NIA-RI } \\
\text { criteria }\end{array}$ & $\begin{array}{l}\text { Category of } \\
\text { LB pathology } \\
\text { by Consensus } \\
\text { criteria - } 2003\end{array}$ & $\begin{array}{l}\text { Probability: } \\
\text { dementia due } \\
\text { to LB pathology } \\
\text { by Consensus } \\
\text { criteria - } 2005\end{array}$ \\
\hline 1 & - & $A D$ & Mixed DLB, AD and $\mathrm{HiScl}$ & 5 & Moderate & Moderate & Probable & NAp & Neocortical & Intermediate \\
\hline 2 & + & DLB & DLB & 0 & None & None & Normal-1c & None & Neocortical & High \\
\hline 3 & + & DLB & DLB & 3 & None & None & Normal-1c & NAp & Neocortical & High \\
\hline 4 & + & DLB & $\begin{array}{l}\text { Mixed DLB and AD in MTL } \\
\text { and SVD }\end{array}$ & 4 & NS & Moderate & Probable & Intermediate & $\begin{array}{l}\text { Neocortical - } \\
\text { severe }\end{array}$ & High \\
\hline 5 & + & CBD & Mixed DLB and AD & 6 & Significant & Frequent & Definite & High likelihood & Neocortical & Intermediate \\
\hline 6 & - & DLB & DLB & 2 & None & Sparse & Possible & Low likelihood & Neocortical & High \\
\hline 7 & + & DLB & $\begin{array}{l}\text { DLB and focal vascular } \\
\text { pathology }\end{array}$ & 2 & None & None & Normal-1c & None & Neocortical & High \\
\hline 8 & + & DLB & DLB & 1 & NS & none & Normal-1c & NAp & $\begin{array}{l}\text { Neocortical - } \\
\text { severe }\end{array}$ & High \\
\hline 9 & - & $A D$ & $\begin{array}{l}\text { AD and focal SVD and } \\
\text { severe Purkinje cell loss }\end{array}$ & 6 & Significant & Frequent & Definite & High likelihood & None & None \\
\hline 10 & - & $A D$ & $\begin{array}{l}\mathrm{AD} \text { and focal vasculopathy } \\
\text { and occipital microinfarct }\end{array}$ & 5 & Moderate & Frequent & Definite & High likelihood & None & None \\
\hline 11 & + & DLB & $\begin{array}{l}\text { Mixed AD and CAA and } \\
\text { haemorrhagic infarcts }\end{array}$ & 5 & Moderate & Frequent & Definite & High likelihood & None & None \\
\hline 12 & - & DLB & $A D$ and mild SVD & 6 & Significant & Frequent & Definite & High likelihood & None & None \\
\hline 13 & + & DLB & $\begin{array}{l}\text { CBD variant - non-tau } \\
\text { balloon cell degeneration } \\
\text { and subcortical tangle } \\
\text { disease }\end{array}$ & 2 & None & Moderate & Probable & NAp & None & None \\
\hline 14 & + & DLB & $A D$ & 5 & $?$ & Frequent & Definite & High likelihood & None & None \\
\hline 15 & - & $A D$ & $\begin{array}{l}\text { Minimal neuropathology: } \\
\text { focal SVD and mild LB } \\
\text { pathology and focal } \\
\text { cortical lesions }\end{array}$ & 1 & None & None & Normal-1c & NÄp & Brainstem & Low \\
\hline 16 & - & $A D$ & $\begin{array}{l}A D \text { and } \mathrm{HiScl} \text { and focal } \\
\text { SVD }\end{array}$ & 6 & Significant & Frequent & Definite & High likelihood & None & None \\
\hline 17 & + & DLB & $\begin{array}{l}\text { FTLD (DLDH) and HiScl and } \\
\text { nigral degeneration }\end{array}$ & 0 & None & None & Normal-1c & None & None & None \\
\hline 18 & - & DLB & $A D$ & 5 & Significant & Frequent & Definite & High likelihood & None & None \\
\hline 19 & + & DLB & $\begin{array}{l}\text { Mixed } A D \text { and } C A A \text { and } \\
\text { focal vascular pathology }\end{array}$ & 6 & Significant & Moderate & Probable & NAp & None & None \\
\hline 20 & - & $A D$ & $\begin{array}{l}\text { Mixed AD and SVD and } \\
\text { multiple microinfarcts }\end{array}$ & 5 & Moderate & Frequent & Definite & High likelihood & None & None \\
\hline
\end{tabular}

AD, Alzheimer's disease; CAA, cerebral amyloid angiopathy; CBD, cortico-basal degeneration; CERAD, Consortium to Establish a Registry for Alzheimer's Disease; DLDH, dementia lacking distinctive histology; DLB, dementia with Lewy bodies; EPF, extrapyramidal features; FTLD, frontotemporal lobar degeneration; HiScl,

hippocampal sclerosis; LB, Lewy body; MTL, medial temporal lobe; NAp, not applicable; NFT, neurofibrillary tangle; NIA-RI, National Institute on Aging and Reagan Institute; NS, not significant; SVD, small vessel disease.

*Newcastle and Helsinki criteria.

\section{Ethics approval}

Ethics approval was obtained for the imaging study, and separate ethics approval was obtained from West Essex Health Authority Ethics Committee for the autopsy follow-up.

\section{RESULTS}

Patient demographic characteristics and clinical features at presentation are shown in tables 1 and 2. There was no significant difference between the neuropathologically diagnosed DLB and non-DLB groups with regard to any of the demographic or clinical characteristics, including the frequency of parkinsonian signs, visual hallucinations and clinical fluctuation. One patient with neuropathologically diagnosed $\mathrm{DLB}$ and two neuropathologically diagnosed $\mathrm{AD}$ patients were receiving neuroleptic medication. One neuropathologically diagnosed CBD patient (clinical diagnosis of DLB) was treated with antiparkinsonian medication, as was one clinically and neuropathologically diagnosed DLB patient. The mean time from clinical diagnosis and FP-CIT scan to autopsy was 34 months (range 6-94).

Patients with a neuropathological diagnosis of DLB had significantly lower radioactivity uptake than non-DLB patients in the caudate nucleus $(p<0.01)$ and in the anterior $(p<0.05)$ and posterior putamen $(\mathrm{p}<0.0001)$.
Clinical diagnoses, semiquantitative imaging assessments (using right and left posterior putamen binding), visually rated imaging assessments and neuropathological diagnoses are displayed in table 3. More detailed neuropathological features and diagnostic criteria are summarised in table 4 .

The sensitivity of an initial clinical diagnosis of DLB was $75 \%$ and specificity was $42 \%$. The sensitivity of visually rated scans for making a diagnosis of DLB was $88 \%$ and specificity was $83 \%$. The sensitivity of semiquantitatively analysed scans was $88 \%$ and specificity was $100 \%$. Using the alternative semiquantitative method where an abnormal scan only needed low posterior putamen binding on one side, sensitivity was 100\% and specificity was $92 \%$ (case No 19, PM diagnosis AD with CVD, had an abnormal scan).

During follow-up of the patients, the clinical diagnoses were changed in three cases; in three further cases with initial diagnoses of DLB the diagnosis was modified from probable to possible. The sensitivity and specificity of the clinical diagnosis of DLB became $88 \%$ and $58 \%$, respectively, but it should be pointed out that these changes in diagnosis were made not just on the basis of further clinical observation of the patients but in the knowledge of the results of their FP-CIT scans, although at the time we did not know how reliable the FP-CIT scans might be. 


\section{DISCUSSION}

The results of this study indicate that FP-CIT SPET scans have good sensitivity and very good specificity for diagnosing DLB. In this series, "dementia not otherwise specified plus abnormal scan" performed considerably better than clinical criteria alone as a means of making the diagnosis of DLB. The FP-CIT semiquantitative measurements performed better than might be anticipated from previous clinico-imaging studies. ${ }^{56}$ In our clinical study, ${ }^{5}$ sensitivity was $52 \%$ and specificity was $94 \%$ (results not shown in the original paper). In a similar study, ${ }^{6}$ sensitivity was $78 \%$ and specificity was $94 \%$. The reason for the better results in our autopsy study is that studies based solely on clinical diagnosis will misclassify a proportion of the patients into incorrect diagnostic categories which leads to a mismatch between scan result and diagnosis.

Compared with previous retrospective and prospective clinicopathological studies, ${ }^{22}$ the sensitivity and particularly the specificity of our clinical diagnosis was poor. There are several possible explanations for this. The clinical diagnosis was made using the Consensus criteria but usually by only one clinician and was not a diagnosis made by the agreement of a panel of three expert clinicians, as was done, for instance, in a Newcastle cohort. ${ }^{9}$ The clinical diagnosis was made at baseline, before patients were scanned. At follow-up, the clinical diagnosis sometimes changed, but for the purpose of this study we used only the baseline diagnosis for analysis of results. The reason for this was that we were particularly interested to gauge how well the FP-CIT imaging technique performed in the initial stages of assessment for dementia. A high proportion of the non-DLB patients were thought to have symptoms and signs characteristic of DLB patients, namely persistent hallucinations, fluctuations and parkinsonian symptoms. On the other hand, one of the DLB patients did not have any core features of DLB at baseline (case No 1) and two DLB patients did not have any parkinsonian symptoms (case Nos 1 and 6). In cases such as these, the scan could be particularly helpful. A high proportion of the non-DLB cases had one or more core features of DLB (see tables 2 and 3). This illustrates the difficulty that clinicians face when making a diagnosis of DLB as all the characteristic features of DLB can be present in possible $\mathrm{AD}$ cases.

The majority of the non-DLB cases had $\mathrm{AD}$ and it might appear surprising that there was no significant difference between the DLB and non-DLB groups with regard to hallucinations and signs of parkinsonism. We believe there are three explanations. Firstly, this was a small series. Secondly, the non-DLB group included a case of CBD and a case of FTD, both of which, unsurprisingly, had rigidity. Thirdly, many of the cases were recruited from a diagnostic memory clinic to which they had been referred because of diagnostic difficulty, so they were possibly not typical cases. This may also account for the relatively low accuracy of the clinical diagnosis of $\mathrm{AD}$ in this series.

In this study, our cut-off between normal and abnormal scans was 2 SDs below the mean of the controls, which is slightly more stringent than the $1.5 \mathrm{SD}$ used by O'Brien and colleagues. ${ }^{6}$ In general, a less stringent cut-off would be expected to increase sensitivity but reduce specificity.

Case No 5 was the only DLB patient whose FP-CIT scan (semiquantitative assessment) was in the normal range. The patient was relatively young, with onset of symptoms at 55 years. The presenting symptom was strikingly unilateral, an inability to use the right hand, with apraxia and myoclonus, leading to an initial clinical diagnosis (by RWHW) of CBD. She then developed a rapidly evolving amnesia. Putaminal FP-CIT binding was clearly abnormal on the left side (visually rated as abnormal), but normal on the right side, such that the right plus left putamen binding ratio was within the normal range. At autopsy (note that the right but not the left sided basal ganglia were examined histologically—see methods), there was only mild neuron loss in the substantia nigra with mild $\alpha$ synuclein pathology, and widespread tangle/neuritic pathology in both the putamen and caudate revealed by AT8 tau immunohistochemistry and Gallyas silver staining. The autopsy did show diffuse neocortical LB-type pathology, and so the patient can be classified as a DLB case, but there was severe AD pathology such that the retrospective likelihood of a clinical DLB syndrome was "intermediate" according to the DLB consortium's third report. ${ }^{21}$ Thus the semiquantitatively assessed scan result represents a false negative. (Using the alternative semiquantitative method, based on a unilateral analysis, the scan was abnormal.)

Assessing scans on the basis of abnormality on one side (whichever side is more abnormal) increased sensitivity but reduced specificity. The typical abnormality in DLB is bilateral loss of binding, mainly in the putamen but also in the caudate nuclei. Generally there is less asymmetry than in Parkinson's disease (PD). ${ }^{23}$ Case No 11 had strictly unilateral loss of putamen binding and so had to be judged visually as abnormal for the purpose of our study even though the appearances were not typical of DLB. The semiquantitative bilateral putamen binding ratio was in the normal range. Autopsy showed extensive vascular disease, which involved the substantia nigra and putamen, accounting for the scan abnormality. Ideally, FPCIT scans should always be evaluated with good quality structural imaging available.

We are aware of reports from only two other groups which have correlated functional imaging and autopsy findings in DLB. ${ }^{724}$ In one, ${ }^{24}$ positron emission tomography (PET) scans of patients with an autopsy diagnosis of DLB or AD were examined retrospectively. Glucose hypometabolism of the occipital cortex, particularly of the primary visual cortex, distinguished DLB from AD with 90\% sensitivity and 80\% specificity. Strictly speaking, none of the "DLB" cases had a clinical diagnosis of DLB but rather had PD with dementia (three cases), and the rest of the patients had a clinical diagnosis of $\mathrm{AD}$. On this evidence, FP-CIT has comparable sensitivity but better specificity than occipital cortex metabolism measured by PET. The other study, ${ }^{7}$ using a functional imaging technique similar to FP-CIT SPECT (dihydrotetrabenazine PET), reported just three cases with autopsy diagnoses of DLB (two cases) and AD (one case), with scan abnormalities in keeping with the autopsy diagnoses.

An abnormal FP-CIT SPECT scan, as defined in this study, equates to a bilateral lesion of the dopaminergic neurons projecting from the substantia nigra to the striatum, specifically to the putamen. Clearly the commonest cause of this is idiopathic PD. The same abnormality is to be expected, and is indeed found, in PD with dementia. ${ }^{6}$ An abnormal scan is not specific for LB pathology. How reliably it identifies patients with DLB will depend on the population of patients tested. For instance, PD and multiple systems atrophy cannot be distinguished by FP-CIT SPECT. Ordinarily, multiple systems atrophy is not characterised by dementia and so will not be a source of false positives. There are however some (mainly rare) neurological disorders which might be expected to give "false positive" results, reducing the specificity of the test. These might include vascular parkinsonism with dementia, and other forms of parkinsonism with dementia such as CBD, progressive supranuclear palsy, frontotemporal dementia with parkinsonism linked to chromosome 17 and Creutzfeldt-Jakob disease. In some of these conditions the main striatal dopaminergic lesion is however postsynaptic rather than presynaptic. Multiple pathologies represent another important possible cause of false 
positive scan results. PD is common. Incidental or presymptomatic nigral LB disease at autopsy is also not uncommon. It must be possible for patients to have, for instance, $\mathrm{AD}$ and also have PD or incidental nigral LB disease without having diffuse LB disease. Autopsies of such cases have been reported. ${ }^{25}$ In such cases, FP-CIT binding in the caudate would be expected to be relatively preserved ${ }^{23}$ but both visual and semiquantitative rating of putaminal binding would give an abnormal scan result. Widespread use of FP-CIT scans in very large numbers of patients with unselected dementia would be expected to generate a number of false positive results. It goes without saying that scanning is no substitute for careful clinical assessment of patients.

What about false negatives? The rationale of the abnormal scan as a test result which supports a diagnosis of DLB is based on the premise that patients with dementia caused by cortical LB pathology will have significant nigrostriatal pathology even if clinical parkinsonism cannot be detected. If there was a group of patients who had extensive cortical LB pathology without nigrostriatal involvement, they would have false negative FPCIT scans. Isolated cases of cortical LB pathology without brainstem LB pathology have been described ${ }^{26}$ but are rare. The majority of reports emphasise that cases with cortical LB pathology invariably also have substantia nigra LB pathology. ${ }^{12} 2728$ It has been suggested that in cases of DLB with cerebral cortex predominant LB pathology, the progression of LB pathology is from the cerebrum to the brainstem. ${ }^{28}$ If that is correct it might be possible for such individuals to have dementia due to cortical LB pathology at a time when their substantia nigra LB pathology was insufficiently advanced to give rise to a positive FP-CIT scan.

The above reservations notwithstanding, our conclusion from this study is that FP-CIT SPECT scanning performed considerably better than clinical criteria as a means of supporting the diagnosis of DLB in patients with dementia. It correlated very well with the presence of LB pathology at autopsy, even with a gap of nearly 3 years, on average, between scan and autopsy (see table 2). It clearly supports the recent change made in the Revised clinical criteria for the diagnosis of $D L B^{21}$ which now includes "low dopamine transporter uptake in the basal ganglia demonstrated by SPECT imaging" as a "suggestive feature" for DLB.

\section{ACKNOWLEDGEMENTS}

We are grateful to the patients and their relatives who took part in the study. We would also like to thank Dr Svetislav Gacinovic from the Department of Nuclear Medicine at University College Hospital, Mr Kenneth Connolly from the Pathology Department at Princess Alexandra Hospital, Mr Bill McMeekin and staff from the Neuropathology Department, Newcastle General Hospital, and Ms Jean Dawes from the Newcastle Brain Tissue Resource.

\section{Authors' affiliations}

Zuzana Walker, Durval C Costa, Gill Livingston, Cornelius L E Katona, University College London and Royal Free Hospitals, London, UK Evelyn Jaros, Robert Perry, Department of Neuropathology, Newcastle General Hospital, UK

Rodney W H Walker, Barts and The London NHS Trust, London, UK

Lean Lee, North Essex Mental Health Partnership NHS Trust, UK

Paul G Ince, University of Sheffield, Sheffield, UK

Ian McKeith, Institute for Ageing and Health, University of Newcastle upon Tyne, Newcastle upon Tyne, UK

The study was supported by grants from Amersham Health and Novartis.

Competing interests: Dr Zuzana Walker, Professor Durval Costa and Professor lan McKeith have received consultancy fees from GE Healthcare (previously Amersham Health), who also provided the FP-CIT ligand.

\section{REFERENCES}

1 Walker Z, Costa DC, Janssen AG, et al. Dementia with Lewy bodies: a study of post-synaptic dopaminergic receptors with ${ }^{123}$ I-IBZM SPET. Eur J Nucl Med 1997:24:609-14.

2 Donnemiller E, Heilmann J, Wenning GK, et al. Brain perfusion scintigraphy with 99mTc-HMPAO or $99 \mathrm{mTc}-\mathrm{ECD}$ and 123I- beta-CIT single-photon emission tomography in dementia of the Alzheimer-type and diffuse Lewy body disease. Eur J Nucl Med 1997;24:320-5.

3 Walker Z, Costa DC, Ince P, et al. In vivo demonstration of dopaminergic degeneration in dementia with Lewy bodies using ${ }^{123}$ I-FP-CIT and SPET. Lancet 1999:354:646-7.

4 Hu XS, Okamura N, Arai H, et al. 18F-fluorodopa PET study of striatal dopamine uptake in the diagnosis of dementia with Lewy bodies. Neurology 2000;55:1575-7.

5 Walker Z, Costa DC, Walker RWH, et al. Differentiation of dementia with Lewy bodies from Alzheimer's disease using a dopaminergic presynaptic ligand. J Neurol Neurosurg Psychiatry 2002;73:134-40.

6 O'Brien JT, Colloby S, Fenwick J, et al. Dopamine transporter loss visualized with FP-CIT SPECT in the differential diagnosis of dementia with Lewy bodies. Arch Neurol 2004;61:919-25.

7 Gilman S, Koeppe RA, Little R, et al. Striatal monoamine terminals in Lewy body dementia and Alzheimer's disease. Ann Neurol 2004;55:774-80.

8 Colloby SJ, O'Brien JT, Fenwick JD, et al. The application of statistical parametric mapping to 123I-FP-CIT SPECT in dementia with Lewy bodies, Alzheimer's disease and Parkinson's disease. Neuroimage 2004;23:956-66.

9 McKeith IG, Ballard CG, Perry RH, et al. Prospective validation of consensus criteria for the diagnosis of dementia with Lewy bodies. Neurology 2000:54:1050-8.

10 Lopez OL, Becker JT, Kaufer DI, et al. Research evaluation and prospective diagnosis of dementia with Lewy bodies. Arch Neurol 2002;59:43-6.

11 McKhann G, Drachman D, Folstein MF, et al. Clinical diagnosis of Alzheimer's disease: Report of the NINCDS-ADRDA Work Group under the auspices of the Department of Health and Human Services Task Force on Alzheimer's disease. Neurology 1984;34:939-44.

12 McKeith IG, Galasko D, Kosaka K, et al. Consensus guidelines for the clinical and pathologic diagnosis of dementia with Lewy bodies (DLB): report of the consortium on DLB international workshop. Neurology 1996;47:11113-24.

13 Lavalaye J, Knol RJ, de Bruin K, et al. [123I]FP-CIT binding in rat brain after acute and sub-chronic administration of dopaminergic medication. Eur J Nucl Med 2000;27:346-9.

14 Booij J, Speelman JD, Horstink MW, et al. The clinical benefit of imaging striatal dopamine transporters with [123I]FP-CIT SPET in differentiating patients with presynaptic parkinsonism from those with other forms of parkinsonism. Eur J Nud Med 2001;28:266-72.

15 Mateos JJ, Lomena F, Parellada E, et al. Lower striatal dopamine transporter binding in neuroleptic-naive schizophrenic patients is not related to antipsychotic treatment but it suggests an illness trait. Psychopharmacology (Berl) 2007:191:805-11.

16 Lorberboym M, Treves TA, Melamed E, et al. [123I]-FP/CIT SPECT imaging for distinguishing drug-induced parkinsonism from Parkinson's disease. Mov Disord 2006;21:510-14.

17 Mirra SS, Heyman A, McKeel D, et al. The Consortium to Establish a Registry for Alzheimer's Disease (CERAD). Part II. Standardization of the neuropathologic assessment of Alzheimer's disease. Neurology 1991;41:479-86.

18 Braak H, Braak E. Neuropathological stageing of Alzheimer-related changes. Acta Neuropathol (Berl) 1991;82:239-59.

19 Ball MJ, Murdoch GH. Neuropathological criteria for the diagnosis of Alzheimer's disease: are we really ready yet? Neurobiol Aging 1997; 18(Suppl 4):S3-12.

20 Perry RH, McKeith IG, Perry EK. International Workshop on Dementia with Lewy Bodies (1995), Newcastle upon Tyne E, Dementia with Lewy bodies:clinical, pathological, and treatment issues. Cambridge: Cambridge University Press, 1996.

21 McKeith IG, Dickson DW, Lowe J, et al. Diagnosis and management of dementia with Lewy bodies: third report of the DLB Consortium. Neurology 2005:65:1863-72.

22 McKeith I, Mintzer J, Aarsland D, et al. Dementia with Lewy bodies. Lancet Neurol 2004;3:19-28.

23 Walker Z, Costa DC, Walker RW, et al. Striatal dopamine transporter in dementia with Lewy bodies and Parkinson disease: a comparison. Neurology 2004;62:1568-72.

24 Minoshima S, Foster NL, Sima AA, et al. Alzheimer's disease versus dementia with Lewy bodies: cerebral metabolic distinction with autopsy confirmation. Ann Neurol 2001;50:358-65.

25 Gibb WR, Mountioy CQ, Mann DM, et al. A pathological study of the association between Lewy body disease and Alzheimer's disease. J Neurol Neurosurg Psychiatry, 1989;52:701-8.

26 Lindboe CF, Hansen HB. The frequency of Lewy bodies in a consecutive autopsy series. Clin Neuropathol 1998;17:204-9.

27 Gomez-Tortosa E, Newell K, Irizarry MC, et al. Clinical and quantitative pathologic correlates of dementia with Lewy bodies. Neurology 1999:53:1284-91.

28 Yamamoto R, Iseki E, Marui W, et al. Non-uniformity in the regional pattern of Lewy pathology in brains of dementia with Lewy bodies. Neuropathology 2005;25: 188-94. 